\title{
Rapid Identification of Saber Steel Flexibility, Microhardness, and Chemical Composition by Analyzing Electromagnetic Signatures
}

\author{
Lucas Braddock Chen \\ West Coast Fencing Academy, US Fencing, Monrovia, USA
}

Email address:

Braddock9955@gmail.com

\section{To cite this article:}

Lucas Braddock Chen. Rapid Identification of Saber Steel Flexibility, Microhardness, and Chemical Composition by Analyzing Electromagnetic Signatures. American Journal of Sports Science. Vol. 8, No. 2, 2020, pp. 29-32. doi: 10.11648/j.ajss.20200802.11

Received: March 18, 2020; Accepted: April 3, 2020; Published: April 17, 2020

\begin{abstract}
Modern fencing sabers are made of composite steel alloys. Although there are regulations standardizing blade dimensions and stiffness, fencers often find blades having variable flexibility and tensile strength from vendor to vendor and, occasionally, from batch to batch of production. Due to the absence of an objective test to assess blade quality, fencers often resort to testing blades with simple visual or physical whip tests. These manual blade assessments, however, are unsatisfactory due to subjective inconsistencies as well as unreliability due to similar blade weights, colors, and appearances. The ability to properly and accurately test blade quality is of utmost importance, not only for competition, but more importantly, to prevent injuries which have occurred as a result of broken blades. Traditional industrial methods of steel alloy identification involve spectrometry or machine tensile strength assessments, which are both destructive to the blade and impractical in a competition arena. We previously reported a method to use the smartphone magnetometer to differentiate fencing steel alloys. This manuscript now demonstrates the correlation between electromagnetic signatures to the flexibility, microhardness and chemical composition of steel blades.
\end{abstract}

Keywords: Fencing, Saber, Steel, Alloy, iPhone, Magnetometer, Magnetism

\section{Introduction}

Soldiers have long utilized fencing and other types of sword drills as preparation for combat. Despite its long history, the sport of fencing did not become standardized until its classification as an Olympic sport in the 1896 Athens Olympic Games, and the equipment blade also became regulated [1]. Nowadays, all fencing weapons are made with carbon-tempered steel [2].

Steel is primarily an iron alloy containing different amounts of carbon $(0.25-2 \%)$, sulfur $(<0.05 \%)$, phosphorus $(<0.04 \%)$, and silicon $(0.3-0.5 \%)$ [3]. Additional elements such as manganese, nickel, chromium, molybdenum, vanadium, silicon, boron, aluminum, cobalt, copper, cerium, niobium, titanium, tungsten, tin, zinc, lead, and zirconium are also added to the steel alloy to provide specific hardness, flexibility, and other properties [4].

Quality of a fencing blade is important to the athlete, as blades with lower hardness are prone to corrosion and deformity, while excessive stiffness results in injury and snapped blades. Despite the importance of blades, however, it is currently impossible for a fencer to easily differentiate with precision between blades of different compositions, since all blades have similar appearances, feel and physical attributes. Fencers rely upon brand labelling, but any brand, however famous or expensive, can have both good and inferior batches of steel production runs.

The traditional industrial methods of steel identification, such as appearance test (color and machine marks), spark test (grinding steel to generate sparks of different color and quality as a reflection of steel composition), and the Rockwell, Brinell or Vickers tests (estimation of steel hardness by measuring the depth or width of indentation from a pounding machine), are subjective and limited. Modern industrial tests are more accurate, but require expensive and cumbersome equipment, such as X-ray fluorescence and atomic emission spectrometry or laserinduced breakdown spectrometers [5]. We previously 
proposed a rapid method of steel identification based on their magnetic properties [6]. Elements such as iron, nickel, cobalt, gadolinium, and dysprosium possess magnetic properties; steel alloys of different grades and quality contain different ratios of these elements, and thus each blade possesses a different magnetic profile (strength and direction of maximum magnetic field) [7]. In addition, since magnetic properties can change while conducting electricity (electromagnetism), each blade demonstrates additional magnetic properties at different levels of electricity due to differences in electrical conductivity (dynamic magnetic property). By measuring the magnetic profiles, a fencer can readily differentiate between, or confirm, different blade compositions.

The ability to quantify magnetism has become more widely available since the arrival of smartphones, since all modern smartphones contain an embedded magnetometer as a part of their internal compass [8]. In this manuscript we demonstrate the correlation between blade magnetic properties and steel blade flexibility, microhardness, and composition.

\section{Material and Methods}

\subsection{Physical Steel Assessment}

All saber blades were purchased brand new from two standard equipment vendors. Each blade was subjected to a flexibility and a hardness test, all performed by Testing Engineers, Inc (San Leandro, CA). The stiffness of the blade was assessed using the Cantilever Deflection Test with specifications detailed in the USA Fencing Rules published June 2018 [9]. Height measurement of each blade tip was recorded, then a 200 gram weight was attached, and the height of tip was recorded again. The test value was taken as the difference between the two heights. The cantilever test was performed using 36" Starrett C305R-36 S/N 44325, TORBAL AD500 S/N 105001062 equipment, with calibration traceable to $\mathrm{N} \sim 1 \mathrm{ST}$. The hardness test was performed by means of the Vickers Microhardness Indentation Test, using a 500 gram load and Leco Microhardness Model: FM-IE S/N" FM1 I 19 equipment.

\subsection{Chemical Steel Assessment}

Each blade was subjected to chemical analysis as performed by Testing Engineers, Inc (San Leandro, CA) using optical emission spectroscopy (OES) and inductively coupled plasma emission spectroscopy (ICP) with the protocols of OES Element SOP 2.02 Rev. 23 for 1812 and S2000, and ICP Element SOP $17.00 \mathrm{Rev} 21$ for DYN. Carbon and Sulfur by combustion per Element SOP 7.00 Rev. 18

\subsection{Electromagnetism Assessment}

The protocol for measuring saber blades' magnetism using the smartphone magnetometer was previously published [10]. Each saber blade was mounted on a rubber insulator to preserve circuit integrity (Rubber Vise Clamp, Amazon, Seattle, WA). Blades were tested without the metal bell guard in order to minimize magnetic interference. A phone stabilizer (Adjustable Universal Gooseneck Smartphone Stand, Entronik, Brooklyn, NY) was used to hold the smartphone (iPhone, XS Max, Apple, Cupertino, CA) running iOS 12.4. Magnetism was measured by the software app Magnetscape 2.0 (App by Toon, Osaka, Japan).

In order to induce dynamic electromagnetism of the blade, an electric circuit was created using a 6-Volt Lantern Battery (Eveready, St. Louis, MO). Circuit was constructed using Alligator clip leads with 18 standard wire gauze stranded copper wires and vinyl sheath (RadioShack, Fort Worth, TX), with multimeter vpro850L, (WeePro, Amazon, Seattle, WA) as the amp meter, and Resistance Substitution Box Model RS-400 (Elenco Electronics, Wheeling, IL) as the variable resistance.

\section{Results}

\subsection{Cantilever Flexibility Deflection Test}

Two regulation saber blades were purchased from each of the two equipment vendors, and all four blades were subjected to the same deflection test (Table 1). Blades from Vendor A deflected by $6.40 \mathrm{~cm}$ and $6.12 \mathrm{~cm}$; blades from Vendor B deflected by $6.55 \mathrm{~cm}$ and $6.86 \mathrm{~cm}$.

\subsection{Vickers Microhardness Indentation Test}

Same two regulation saber blades from each equipment vendor were assessed via the Vickers Microhardness Indentation Test using a 500 gram load (Table 2). Measurements were taken at two same points on each blade. One point of measurement was taken at the "center," which for the purpose of the study was defined as $30 \mathrm{~cm}$ from the hilt (where the blade emerges from the guard), and one measurement was at the "tip," which for the purpose of the study was defined as $1 \mathrm{~cm}$ from the tip end of the blade. Five microhardness indentation tests were performed at each of the two measurement points on each blade, and the average taken at each point was recorded into the table. The microhardness of each blade varied at both points of measurements. Center hardness varied from $641 \mathrm{HV}$ (Vickers Hardness) to $687 \mathrm{HV}$. Tip hardness varied from $536 \mathrm{HV}$ to $647 \mathrm{HV}$. The bottom row of the table shows the average microhardness of each blade (average of the Center and Tip measurements).

\subsection{Chemical Steel Analysis}

All four blades were analyzed for their chemical composition, and the results are shown as percentage by weight (Table 3). There is significant difference in terms of elemental composition of each steel alloy, with Vendor A blades having minimal carbon, but having cobalt, titanium and aluminum, which are not present in the blades from Vendor B. 


\subsection{Dynamic Magnetic Profile}

All four blades were assessed for both their baseline magnetic properties as well as their electromagnetic properties when charged in electric circuit (Table 4). The level of magnetic strength was recorded as MicroTesla $(\mu \mathrm{T})$, and the bottom row shows the difference between baseline magnetism and induced electromagnetism. Blades from Vendor B showed a greater increase of magnetic field strength when placed in an electric circuit.

\section{Conclusion}

The quality of a fencing blade is of critical importance, both in terms of competition as well as for the safety of the athletes. It has always been difficult for a fencer to precisely identify the quality of blades due to their similar appearances and the absence of field tests. We previously proposed a method to differentiate steel alloys based on their unique magnetic profiles from different metal alloy compositions. We also published the methodology for conducting the magnetic measurements. We now describe the correlation between steel alloy flexibility, microhardness, chemical composition, and magnetic property.

Four brand new competition saber blades from two different vendors were analyzed for this study. The Cantilever Flexibility Deflection Test showed that blades from each vendor had different degrees of flexibility, with Vendor B having more flexible blades. There is also difference of flexibility within each vendor's blades. It is possible that the differences are the result of different production runs. It is also possible that vendors deliberately create blades to different flexibilities to conform to fencers' different preferences and fencing styles. It is important to note that all blades conform to the flexibility standard as set forth by the USA Fencing Guide.
Vickers Microhardness Indentation Test showed that blades from each vendor have overlapping and different degrees of hardness. In addition, each vendor also created different blade styles, with some having uniform microhardness both at mid-shaft and tip, while some have decreased microhardness at the tip. As before, it is possible that different blade styles were created to cater to different athletes.

Chemical analysis showed that the vendors use very different kinds of steel. Vendor A steel had less carbon, phosphorus, silicon, manganese, chromium and copper, while containing more nickel and molybdenum. In addition, Vendor A steel alloy also contained aluminum, cobalt, and titanium, which were all absent from the blades from Vendor B. These differences in chemical composition were reflected in their magnetic profiles. Blades from Vendor A had limited electromagnetic increase when induced inside a circuit (increase of $0.81 \mu \mathrm{T}$ and $0.67 \mu \mathrm{T}$ ), while blades from Vendor $\mathrm{B}$ had a higher jump of electromagnetism $(1.38 \mu \mathrm{T}$ and 1.59 $\mu \mathrm{T}$ ). The increase in electromagnetism is most likely due to the different chemical composition having different electrical conductivity and different paramagnetic properties. The differences of electromagnetic signatures also correspond to the degree of flexibility as determined by the Cantilever Flexibility Deflection Test: higher electromagnetism increase correlates to higher blade flexibility.

We report that electromagnetism as assessed by smartphone magnetometers can differentiate between sabers of different chemical compositions and flexibility. The current study was conducted using one electric source. Further study would benefit from using variable levels of electric currents and thus, different levels of induced electromagnetism to further characterize steel of different composition. A reference library of electromagnetic signatures will allow rapid identification of steel types, which is a study currently underway.

Table 1. Cantilever Flexibility Deflection Test: Two regulation saber blades from each vendor were tested. Height measurement of each tip was recorded before mass application, then a $200 \mathrm{~g}$ weight was attached, and the height of each tip was recorded again. Test value was taken as the difference between the two heights.

\begin{tabular}{lllll}
\hline & Vendor A & & Vendor B & \\
\cline { 2 - 5 } & Blade \#1 & Blade \#2 & Blade \#3 & Blade \#4 \\
\hline Deflection $(\mathrm{cm})$ & 6.40 & 6.12 & 6.55 & 6.86 \\
\hline
\end{tabular}

Table 2. Vickers Microhardness Indentation Test: Two regulation saber blades from each equipment vendor were assessed for microhardness using a 500-gram load. Measurements were taken at the same two points on each blade. One point of measurement was taken at the "center," which was defined as $30 \mathrm{~cm}$ from the hilt, and one measurement was taken at $1 \mathrm{~cm}$ from the tip end of the blade. Five microhardness indentation tests were performed at each of the two measurement points of each blade, and the average taken at each point was recorded into the table.

\begin{tabular}{lllll}
\hline \multirow{2}{*}{ Vickers Hardness (HV)* } & Vendor A & \multicolumn{2}{c}{ Vendor B } \\
\cline { 2 - 5 } & Blade \#1 & Blade \#2 & Blade \#3 & Blade \#4 \\
\hline Microhardness (Center) & 687 & 641 & 655 & 647 \\
Microhardness (Tip) & 647 & 641 & 536 & 647 \\
Average & 667 & 641 & 595.5 & 647 \\
\hline
\end{tabular}

*The Vickers hardness test method indents the test material with a diamond pyramid with a square base and an angle of 136 degrees between opposite faces subjected to a load as measured in kgf. The full load is normally applied for 10 to 15 seconds. The two diagonals of the indentation are measured using a microscope and their average calculated. The area of the sloping surface of the indentation is calculated. The Vickers hardness is the quotient obtained by dividing the kgf load by the square $\mathrm{mm}$ area of indentation. $F=$ Load in kgf; $d=$ Arithmetic mean of the two diagonals, $d 1$ and $d 2$ in mm; HV $=$ Vickers hardness [11, 12] 
Table 3. Chemical Steel Analysis: All four blades were analyzed for their chemical composition. Results are expressed as percentage based on weight (\%).

\begin{tabular}{lllll}
\hline Percentage by Weight & Vendor A & & Vendor B & Blade \#4 \\
\hline $\mathbf{( \% )}$ & Blade \#1 & Blade \#2 & Blade \#3 & 0.59 \\
\hline $\mathrm{C}$ & $<0.01$ & 0.01 & 0.59 & $<0.005$ \\
$\mathrm{~S}$ & $<0.005$ & $<0.005$ & $<0.005$ & 0.017 \\
$\mathrm{P}$ & $<0.01$ & $<0.01$ & 0.013 & 1.75 \\
$\mathrm{Si}$ & 0.05 & 0.05 & 1.76 & 0.73 \\
$\mathrm{Mn}$ & 0.05 & 0.04 & 0.71 & 0.19 \\
$\mathrm{Cr}$ & 0.12 & 0.12 & 0.18 & $<0.01$ \\
$\mathrm{Ni}$ & 18.65 & 18.65 & 0.02 & 0.14 \\
$\mathrm{Mo}$ & 4.95 & 4.85 & $<0.01$ & \\
$\mathrm{Cu}$ & 0.05 & 0.06 & 0.14 & \\
$\mathrm{Sn}$ & & & \\
$\mathrm{Al}$ & 0.1 & 0.1 & & \\
$\mathrm{~B}$ & & & & \\
$\mathrm{Co}$ & 8.8 & 8.8 & & \\
$\mathrm{Ti}$ & 0.68 & 0.67 & & \\
$\mathrm{Ca}$ & & & & \\
$\mathrm{Zr}$ & & & & \\
\hline
\end{tabular}

Table 4. Electromagnetic Assessment: All four blades were assessed for both their baseline magnetic properties as well as their electromagnetic properties when charged in electric circuit [13]. The level of magnetic strength was recorded as MicroTesla ( $\mu$ T) [14, 15]; the bottom row shows the difference between baseline magnetism and electromagnetism.

\begin{tabular}{lllll}
\hline \multirow{2}{*}{ Magnetism in MicroTesla $(\boldsymbol{\mu} \mathbf{T})$} & Vendor A & \multicolumn{2}{c}{ Vendor B } \\
\cline { 2 - 5 } & Blade \#1 & Blade \#2 & Blade \#3 & 41.42 \\
\hline Baseline Magnetism & 41.71 & 41.13 & 42.80 & 41.13 \\
Electromagnetism & 42.52 & 41.80 & 4.38 & 42.72 \\
Difference & 0.81 & 0.67 & 1.59 & 1.38 \\
\hline
\end{tabular}

\section{References}

[1] International Olympic Committee. Fencing: History of Fencing at the Olympics Games. Olympics Studies Centre. March 2015.

[2] AZOM. Advanced Materials in the Sport of Fencing. AZO Materials. March 15, 2001. https://www.azom.com/article.aspx?ArticleID=179.

[3] Science Encyclopedia. Steel: Raw Materials. Downloaded September 18 , 2019. http://science.jrank.org/pages/6483/Steel-Raw-materials.html.

[4] Smith, William F, Hashemi, Javad. Foundations of Material Science and Engineering. McGraw-Hill, 2001.p. 394.

[5] Vericheck Technical Services. Common Metal Detection Methods. December 1, 2016. https://verichek.net/commonmetal-identification-methods.html.

[6] Chen, Lucas. Determining Fencing Blade Quality using Dynamic Magnetic Field Measurements. The United States Trademark and Patent Office, U.S. Serial Number 16/422, 731. May 24, 2019.

[7] Wallulis, Karl. List of Metals That Are Attracted to Magnets. April 17, 2018. https://sciencing.com/list-metals-attractedmagnets-7501815.html.

[8] TechBlog. Using techBASIC to Turn Your iPhone or iPad into a Metal Detector. November 30, 2011. http://www.byteworks.us/Byte_Works/Blog/Entries/2011/11/3 0_Using techBASIC_to_Turn_Your_iPhone_or_iPad_into_a Metal_Detector.html.

[9] USA Fencing Rules, June 2018. https://cdn3.sportngin.com/attachments/document/7c681570818/2018-USA-Fencing-Rules.pdf.

[10] Chen, Lucas. Differentiation of Fencing Blade Alloys Using iPhone Magnetometer. $\mathrm{J}$ Experimental Techniques and Instrumentation. April 2020 (in press).

[11] Vickers Hardness Test. https://www.gordonengland.co.uk/hardness/vickers.htm.

[12] Delfini, Ron. Rockwell, Brinell, and Vickers Metal Hardness Tests: What's the Difference? Engineering Specialties, June 14, 2018. https://www.esict.com/blog/rockwell-brinell-andvickers-metal-hardness/

[13] Korolef, Alexander I. On electromagnetic induction in electric conductors. General Physics. February 22, 2013. https://arxiv.org/abs/1303.0785

[14] Australian Radiation Protection and Nuclear Safety Agency. Measuring Magnetic Fields. 2020. https://www.arpansa.gov.au/understandingradiation/radiation-sources/more-radiationsources/measuring-magnetic-fields

[15] Alvarado, Lucinette. Electromagnetic Fields in the Steel Industry. Iron \& Steel Technology. June 2014, p36-39. 